\title{
Expression of Hepatocyte Markers in Wharton's Jelly Mesenchymal Stem Cells Using Mouse Liver Cell Extract
}

\section{Maryam Borhani-Haghighi $^{1,2^{*}}$, Fatemeh Alipour ${ }^{1}$, Arezou Eshaghabadi ${ }^{1}$}

${ }^{1}$ Shefa Neuroscience Research Center, Khatam Alanbia Hospital, Tehran, Iran

${ }^{2}$ Department of Anatomy, School of Medicine, Tehran University of Medical Sciences, Tehran, Iran

\section{A BSTRACT}

Introduction: Efforts have been taken to find appropriate sources to replace liver transplantation. Wharton's jelly is an unlimited source of stem cells that can be used in cell therapy and tissue engineering. In this study we investigated whether Wharton's jelly-derived mesenchymal stem cells (WJMSCs) could trans-differentiate into hepatocyte in the presence of mouse liver cell-free extract. Materials and Methods: Mesenchymal stem cells (MSCs) were isolated from the umbilical cord. The cells were permeabilized by Sterptolysin $\mathrm{O}$ in the presence of mouse liver cell-free extract for 21 days. To evaluate differentiation and morphological changes, immunostaining for cytokeratin 18 and 19 were performed for differentiated and control cells. Functional assays were done by periodic acid Schiff (PAS) stain. Results: The phenotype of treated MSCs in the presence of liver cell-free extract changed into polygonal cells. Immunostaining demonstrated the expression of cytokeratin 18 and 19 in differentiated cells. Glycogen storage in differentiated cells revealed by PAS staining indicated functional property of differentiated cells. Conclusion: It seems that factors existing in the extract able to trans-differentiate WJMSCs into functional hepatocyte.

"Corresponding Author: Maryam Borhani-Haghighi

E-mail: borhanihm@gmail.com 
بيان نشانگَرهاى كبدى در سلولهاى بنيادى مزانشيمى زله وارتون بند ناف توسط عصارهُ سلول كبد موش مزانشي

\author{
مريم برهانى حقيقى ‘،:ا:، فاطمه علىيور' ، آرزو اسحق آبادى' \\ 'مركز تحقيقات علوم اعصاب شفا، بيمارستان خاتمالانبياء، تهران، ايران

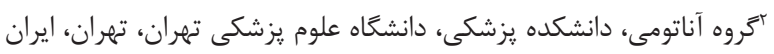

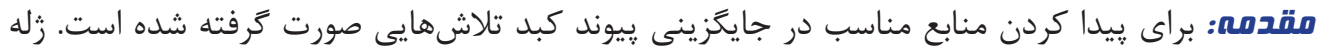

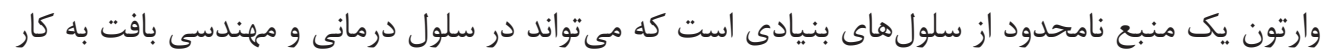

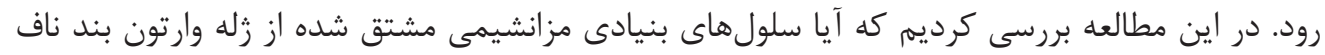

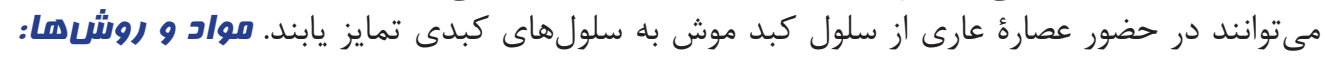

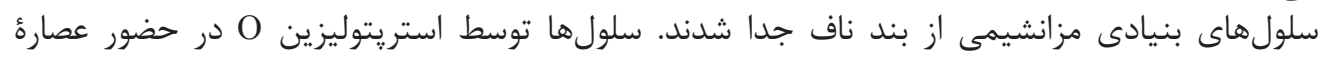

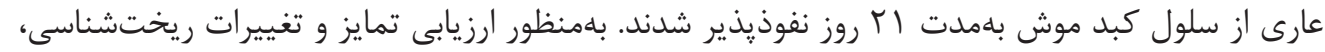

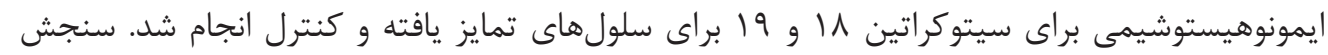

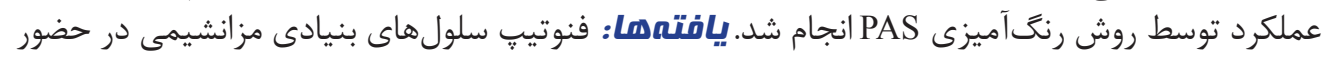

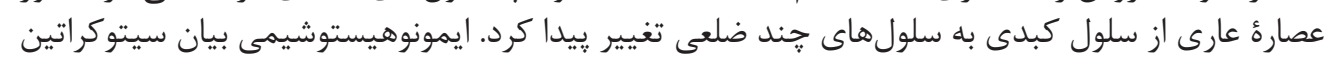

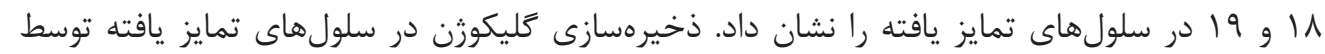

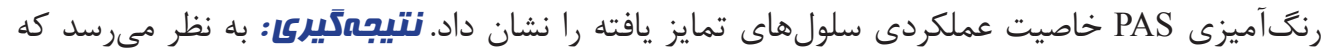

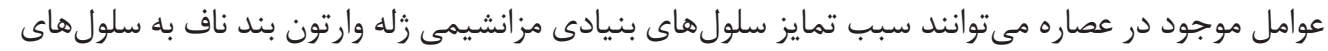
كبدى عملكردى شوند.

كليد وازهها:

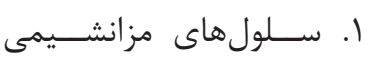

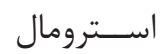

r.

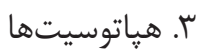

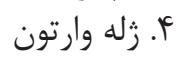

" نويسنده مسئول: مريمم برهانى حقيقى آدرس الكترونيكى: borhanihm@gmail.com 


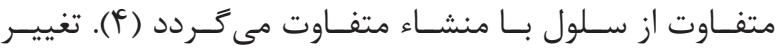

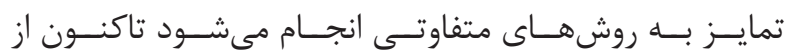

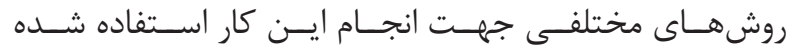

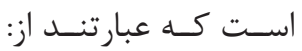
Cloning Nuclear Transfer.)

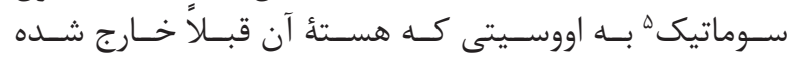

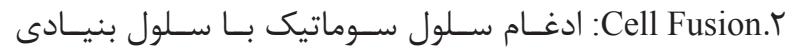

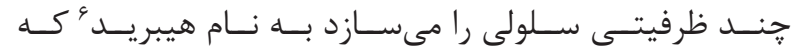

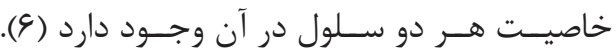
س. Gene Transfection.r

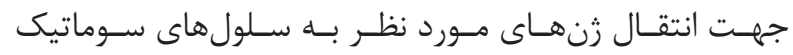

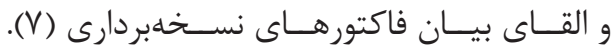

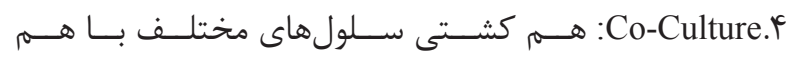

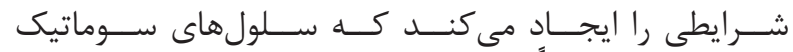

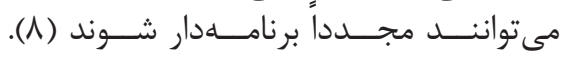

ه. Cell Extract: استفاده از عصارههاى سلولى (9). در ايـن تحقيـق سـعى مـا بر ايـن بود تـا بـا اسـتفاده از عصاره

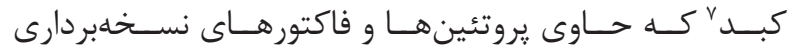

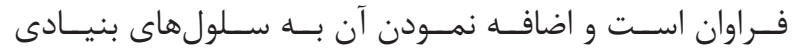

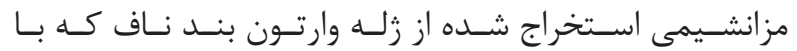

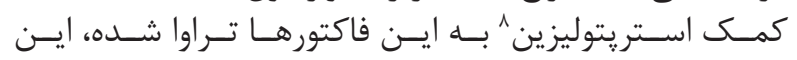

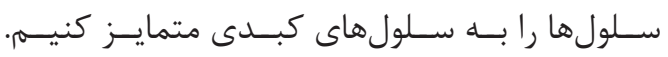

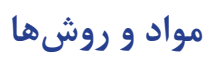

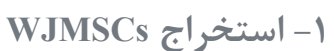

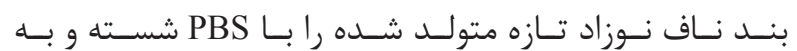

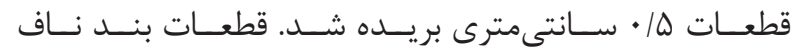

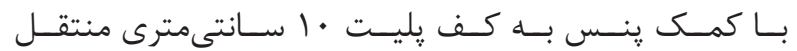

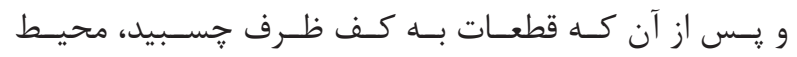

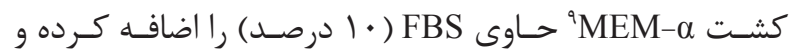

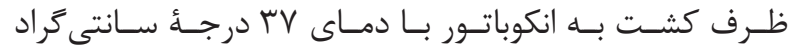

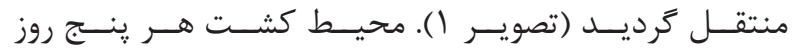

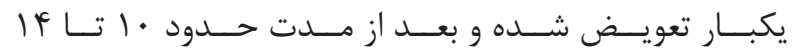

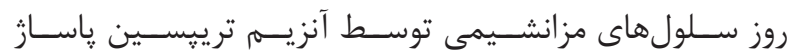

كرديدنــد.
مقدمه

بــر اسـاس آمارهـاى سـازمان بهداشـت جهانـى بيـش از صدها

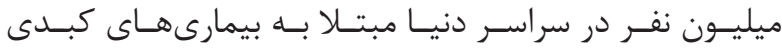

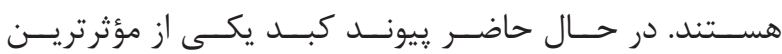

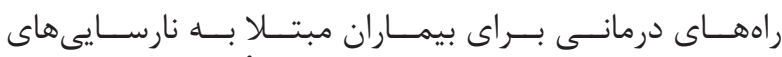

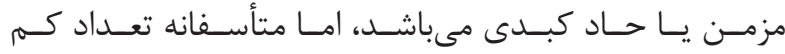

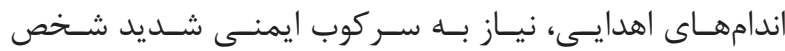

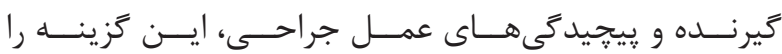

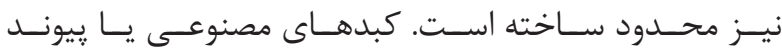

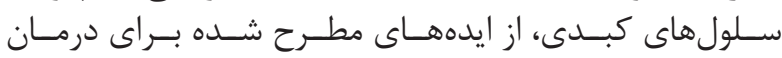

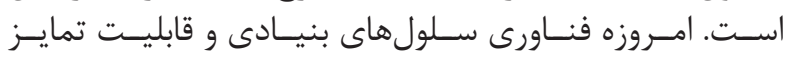

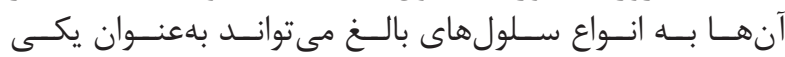

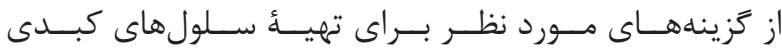

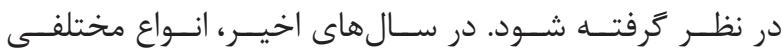

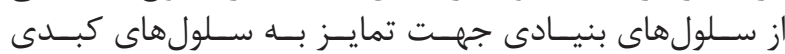

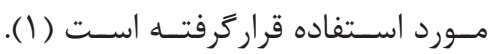

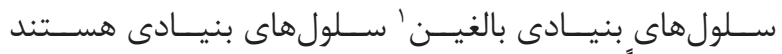

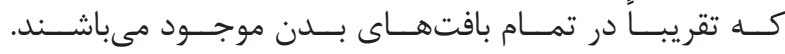

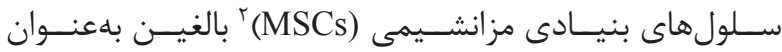

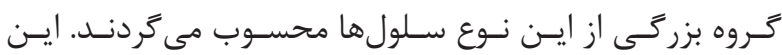

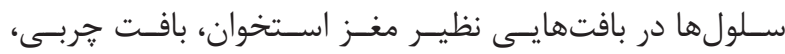

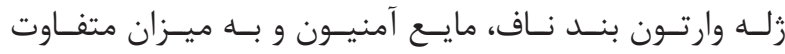

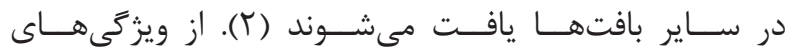

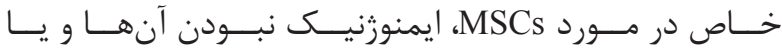

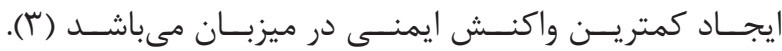

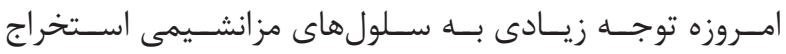

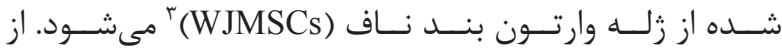

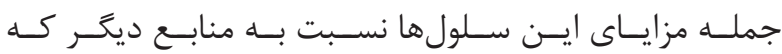

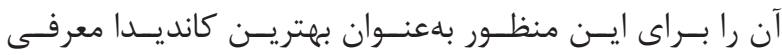

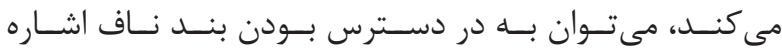

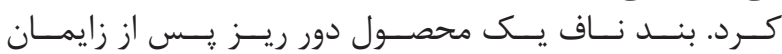

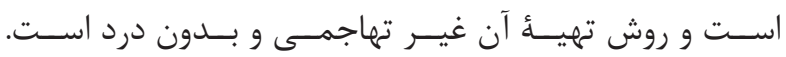

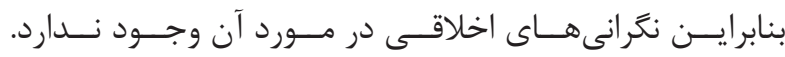

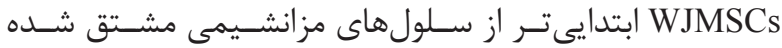

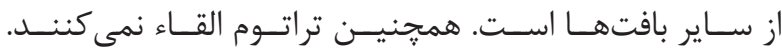

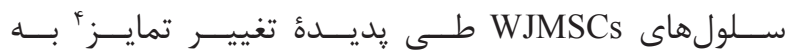

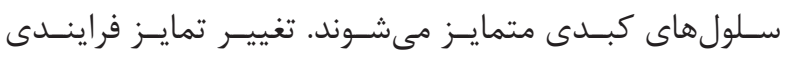

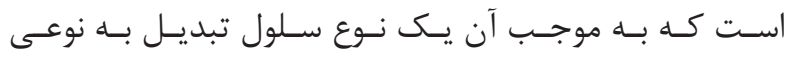
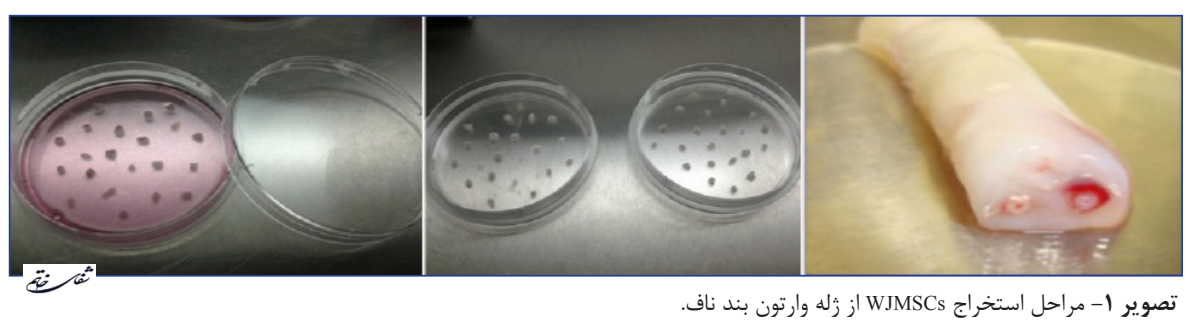

${ }^{1}$ Adult stem cell

${ }^{2}$ Mesenchymal stem cells

${ }^{3}$ Wharton's jelly mesenchymal stem cells

${ }^{4}$ Transdifferentiation

${ }^{5}$ Somatic cell
تصوير ا-مراحل استخراج WJMSCs از زله وارتون بند ناف.

${ }^{6}$ Hybrid

${ }^{7}$ Liver extract

${ }^{8}$ Sterptolysin

${ }^{9}$ Minimum essential medium eagle alpha 
ها- آزمون نفوذيذيرى

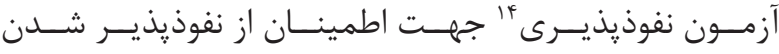

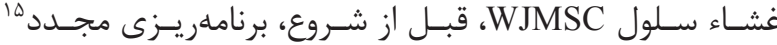

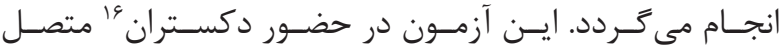

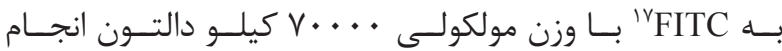

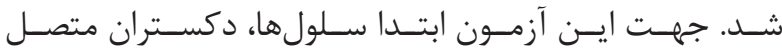

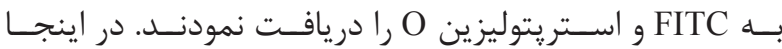

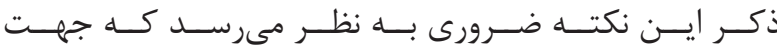

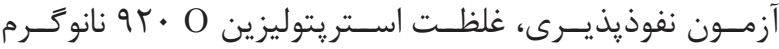

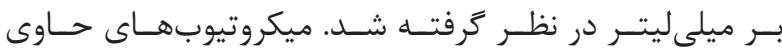

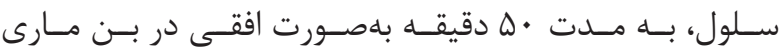

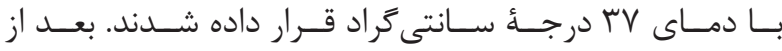

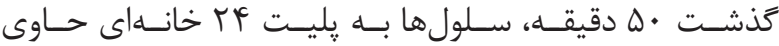

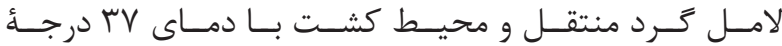

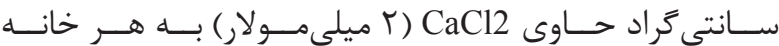

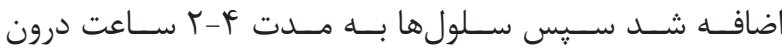

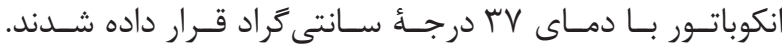

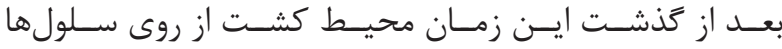

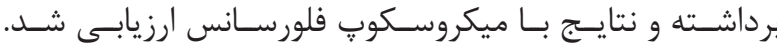

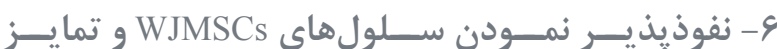

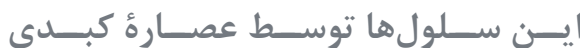

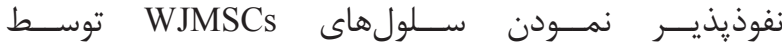

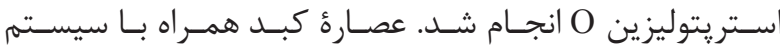

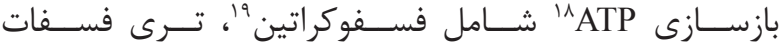

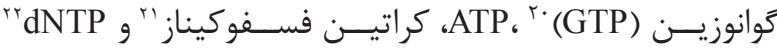

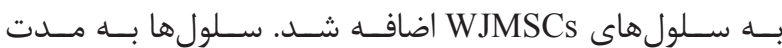

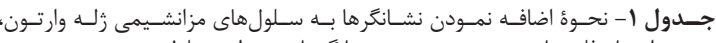

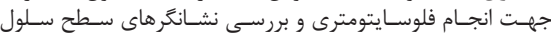

\begin{tabular}{|c|c|c|}
\hline رديف & ليبل & نشالكر \\
\hline 1 & Percp (Peidinin chlorophyll protein complex) / PE (Phycoesythrin) / FITC & CD 34: CD 44: CD 105 \\
\hline r & $\mathrm{PE} / \mathrm{FTTC}$ & CD 144.CD 106 \\
\hline$r$ & FITC & CD 44 \\
\hline$\varphi$ & PE & CD 106 \\
\hline$\Delta$ & Perpp & CD 105 \\
\hline \& & FITC & CD 90 \\
\hline y & FITC / PE & CD 106.CD 44 \\
\hline A & Percy / FITC & CD 44.CD 105 \\
\hline 9 & Perç/ PE & CD 34.CD 105 \\
\hline
\end{tabular}

${ }^{10}$ Hanks' balanced salt solution

${ }^{11}$ Dithiothreitol

${ }^{12}$ Bradford

${ }^{13}$ Optical density

${ }^{14}$ Permeabilization assay

${ }^{15}$ Reprogramming

${ }^{16}$ Dextran

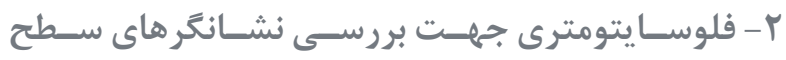

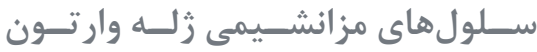

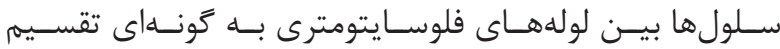

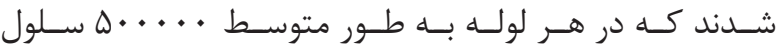

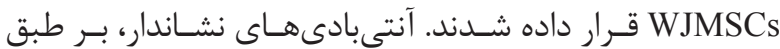

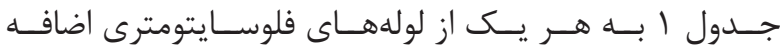

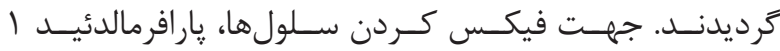

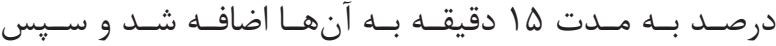

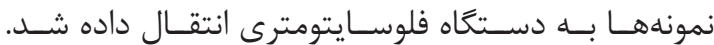

$$
\text { "- به دست آوردن عصارة كبد }
$$

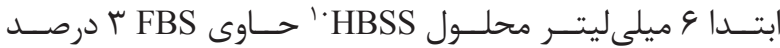

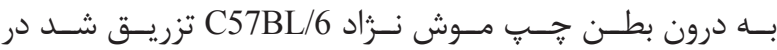

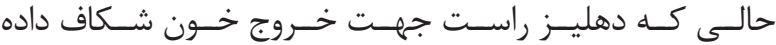

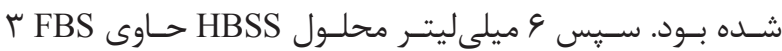

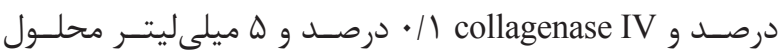

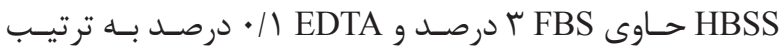

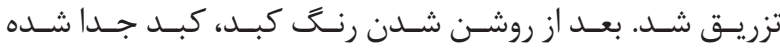

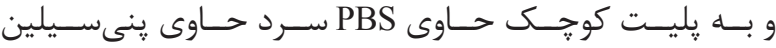

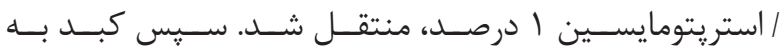

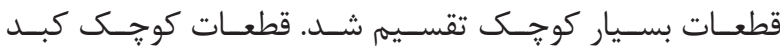

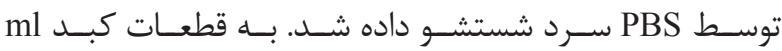

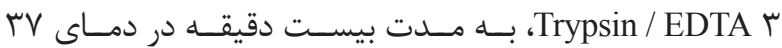

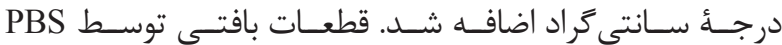

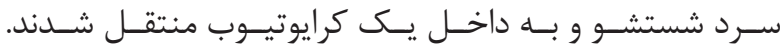

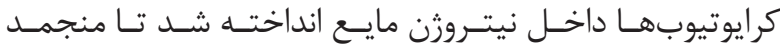

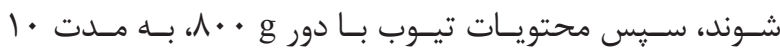

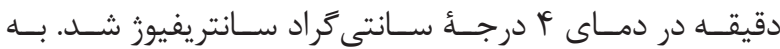

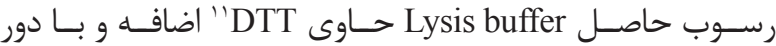

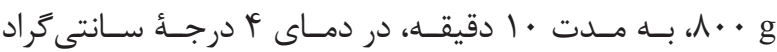

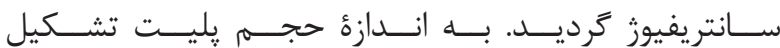
شـده،Lysis buffer حـــاوى

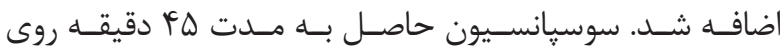

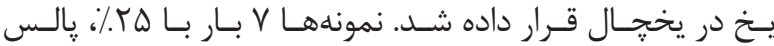

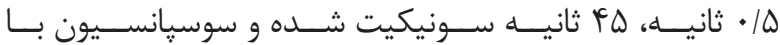

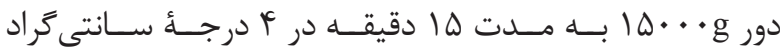

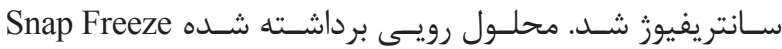

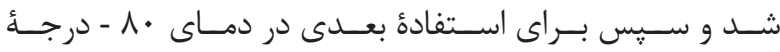

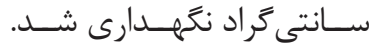

P - اندازهَيرى ميزان ثبروتئين موجود در عصاره

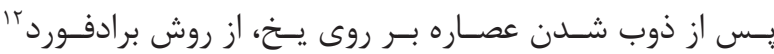

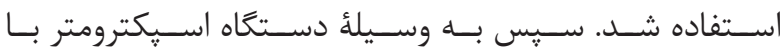

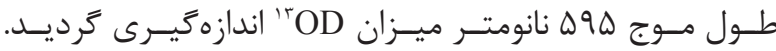

${ }^{17}$ Fluorescein isothiocyanate

${ }^{18}$ Adenosine triphosphate (ATP) regenerating system

${ }^{19}$ Phosphocreatine

${ }^{20}$ Guanosine triphosphate

${ }^{21}$ Creatine phosphokinase

22 Deoxynucleotide 


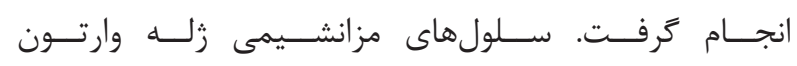

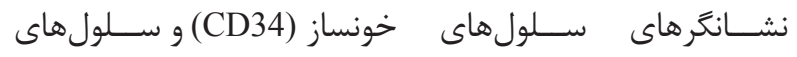

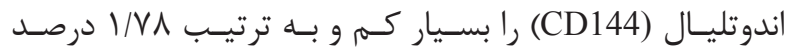

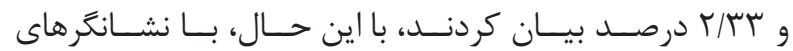

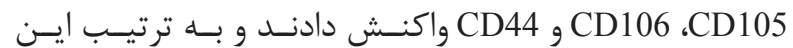

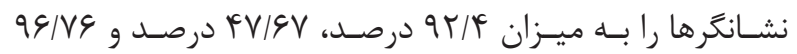
درصـد بيـان كردنــد (نمـودار () ).
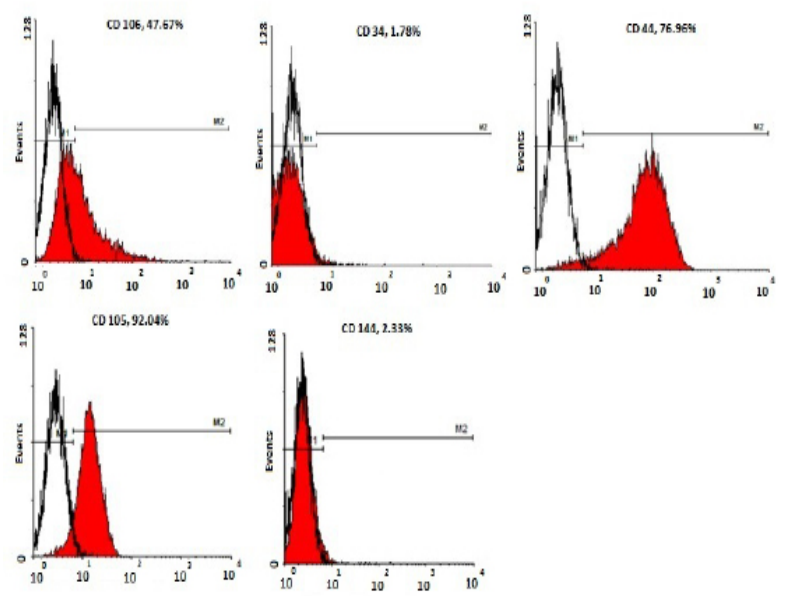

ث

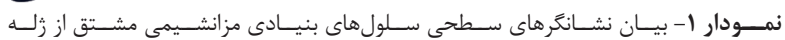

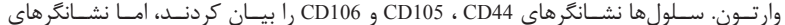

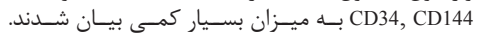

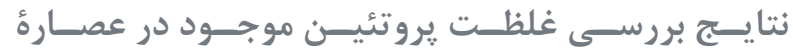

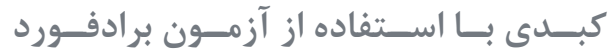

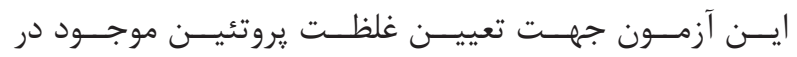

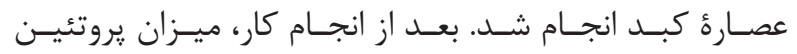

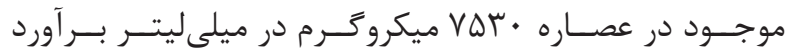

شـد.

نتايج آزمون سمى بودن عصارء كبد

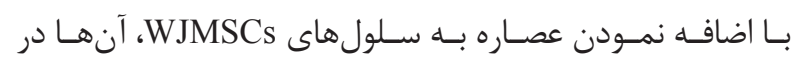

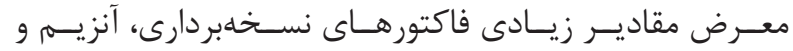

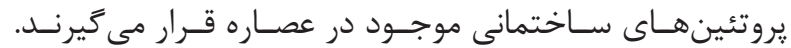

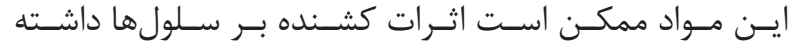

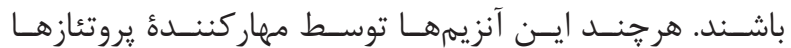

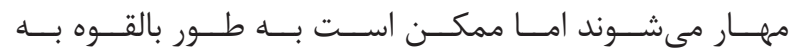

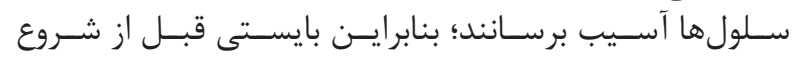

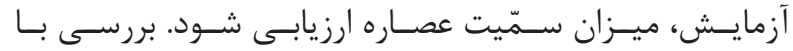

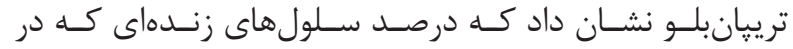

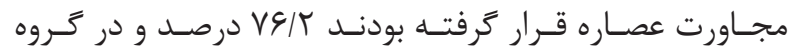

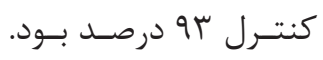

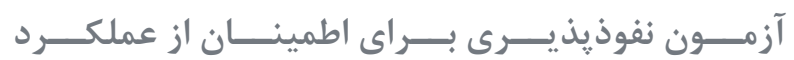

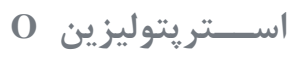

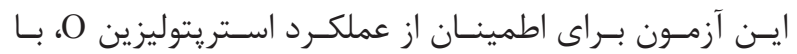

${ }^{23}$ Periodic acid-schiff

${ }^{24}$ Hydrogen peroxide

25 3,3'-diaminobenzidine

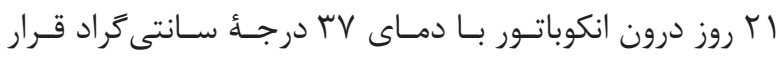

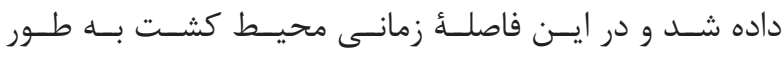

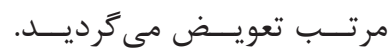
- برسى عملكرد سلول هاى تمايز يافته

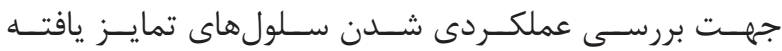

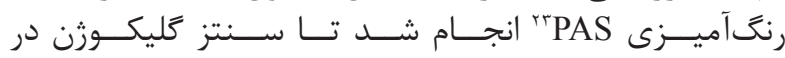

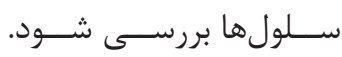

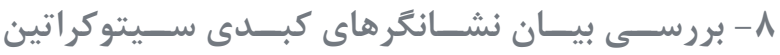

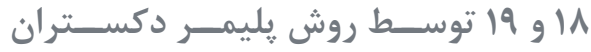

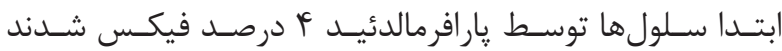

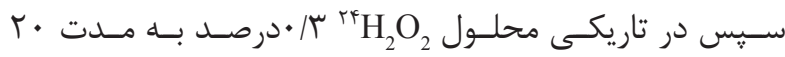

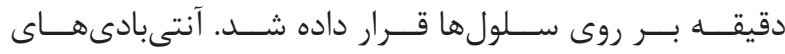

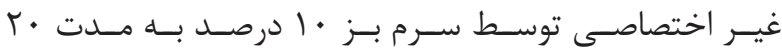

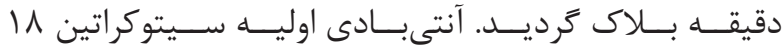

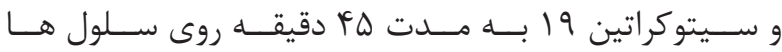

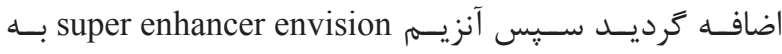

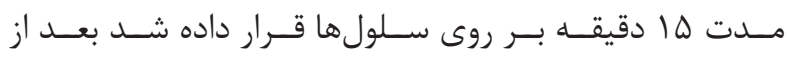

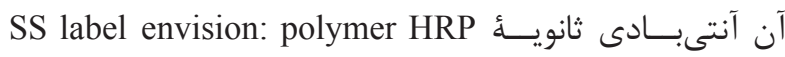

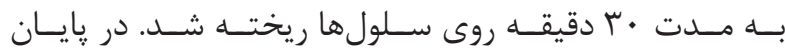

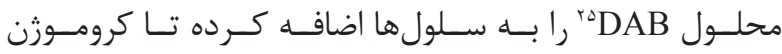

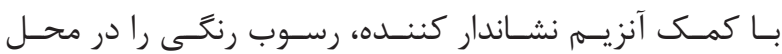

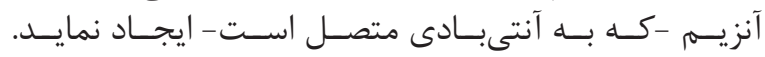

بافتهها

WJMSCs بررسى ريختشناسى سلولهاى

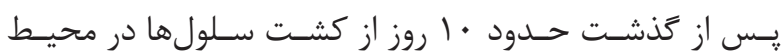

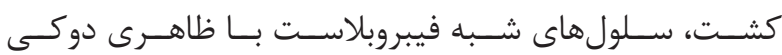

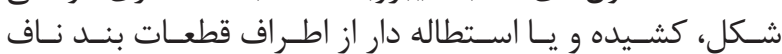

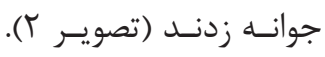

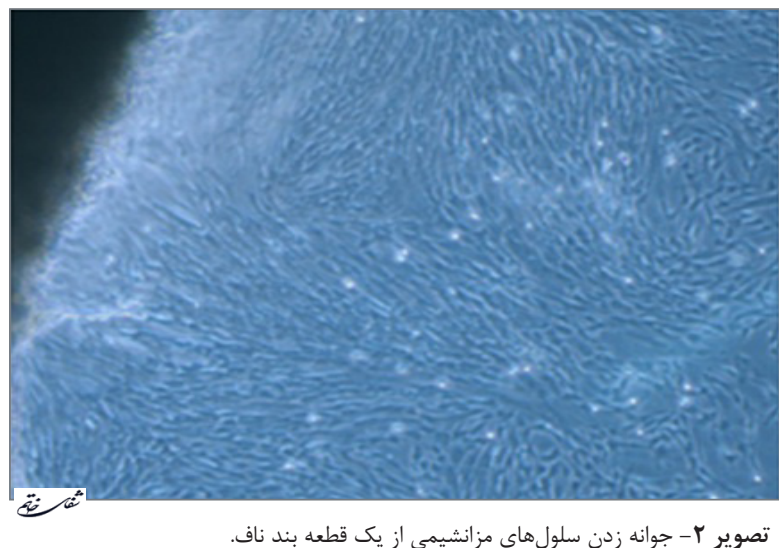

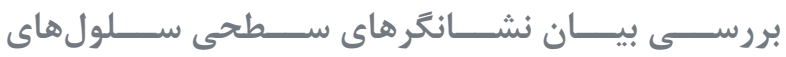
WJMSCs

بلهنظــور بررسـى نشـانخر هاى سـطح سـلولى، فلوسـايتومترى 


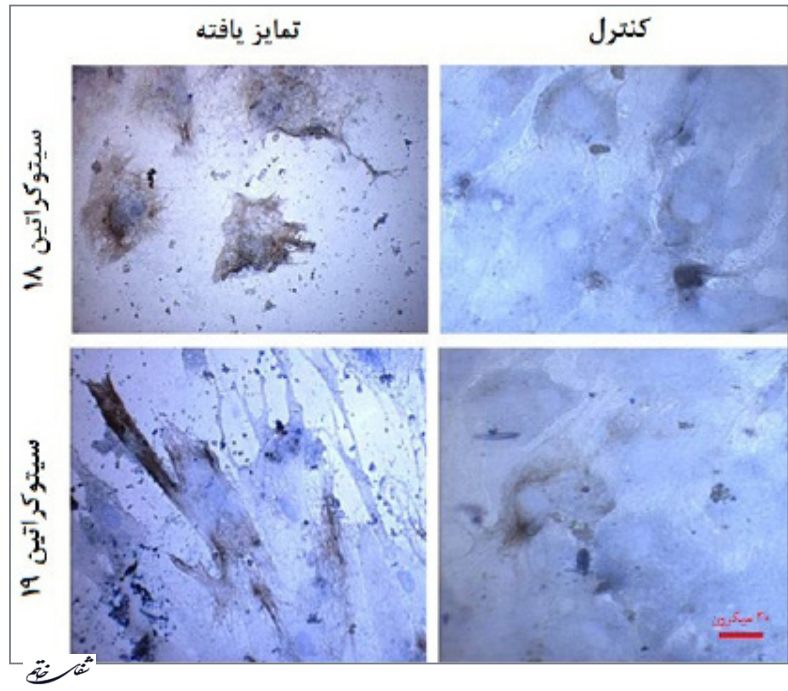

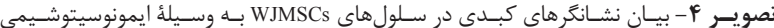

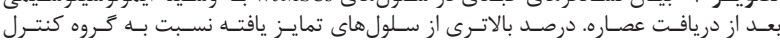

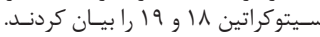

PAS در سـلولهاى WJMSCs بعـد از دريافـت عصـاره

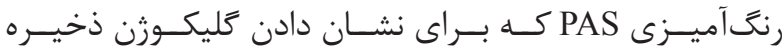

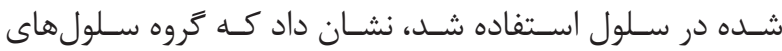

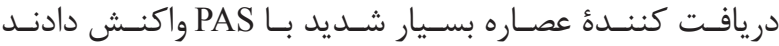

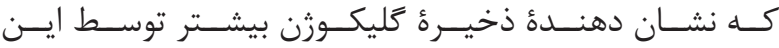

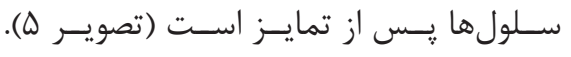

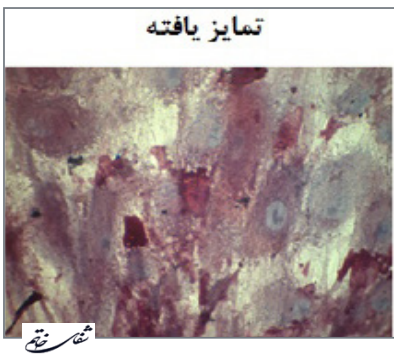
كنترل

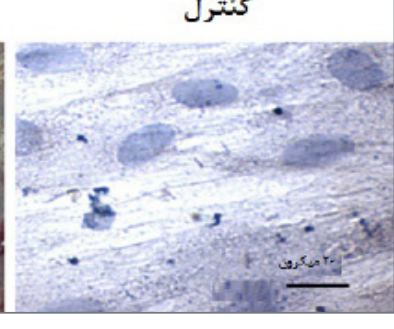

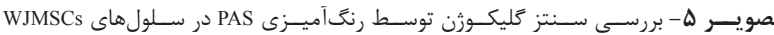

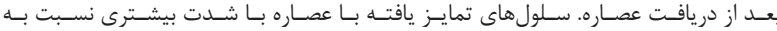

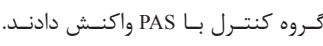

بحث و نتيجه

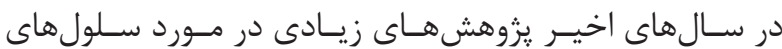

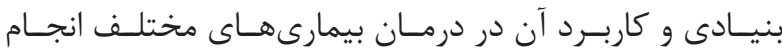

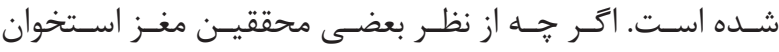

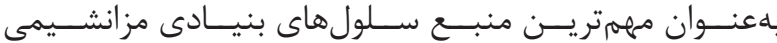

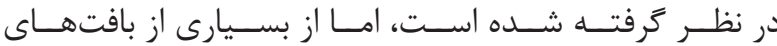

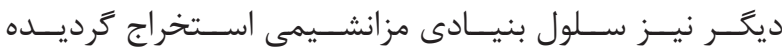

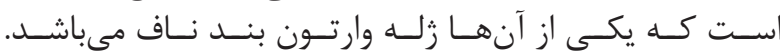

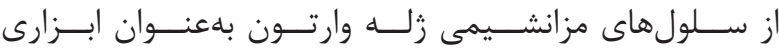

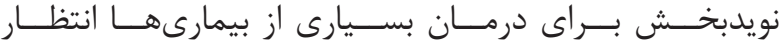

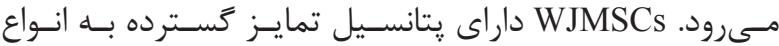

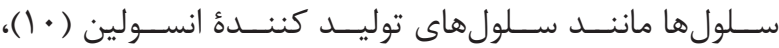

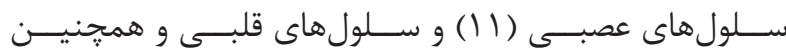

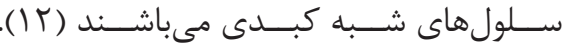

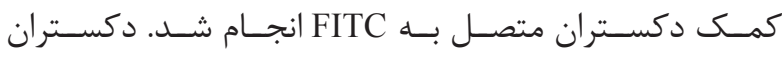

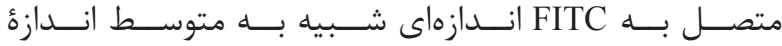

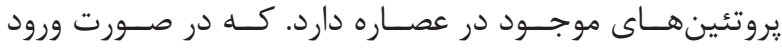

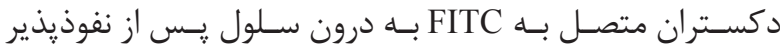

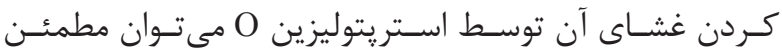

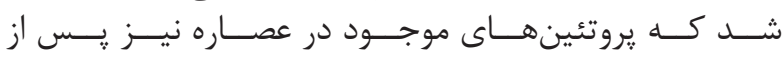

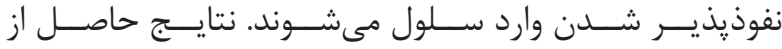

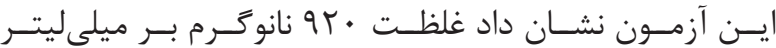

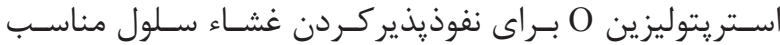

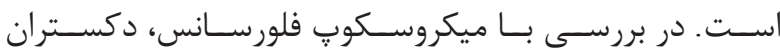

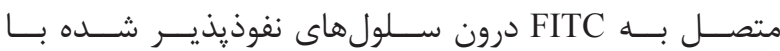

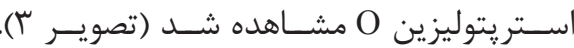

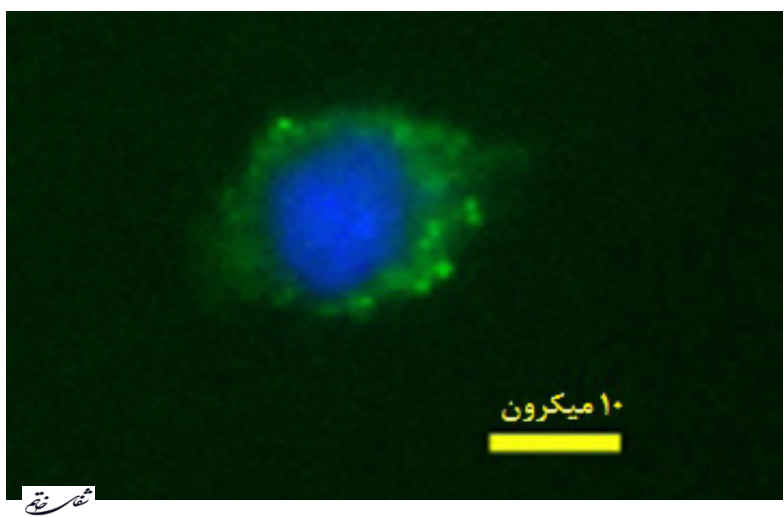

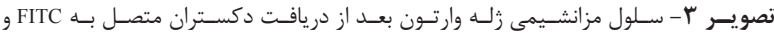

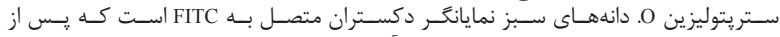

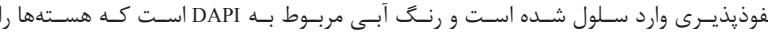

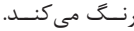

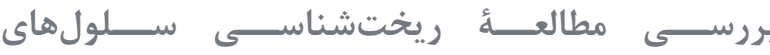

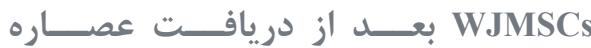

در حالـت طبيعى سـلولهاى WJMSCs كشـيده و اسـتطاله

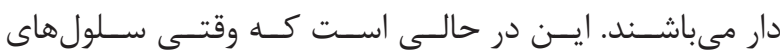

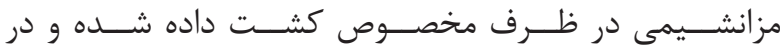

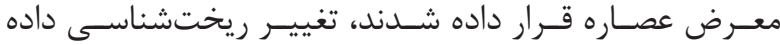

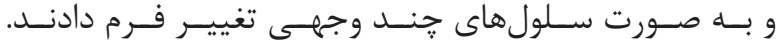

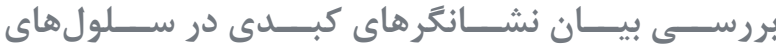

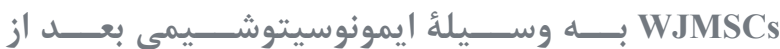
دريافـت عصــاره

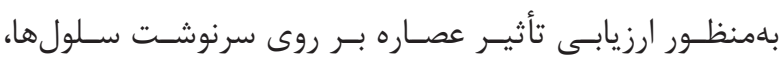

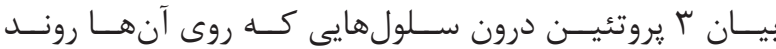

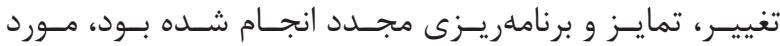

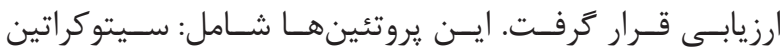

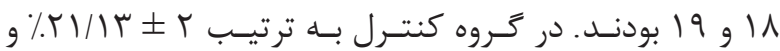

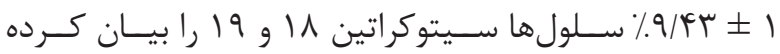

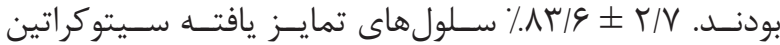

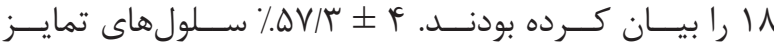

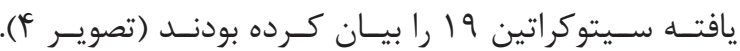

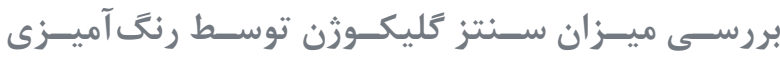




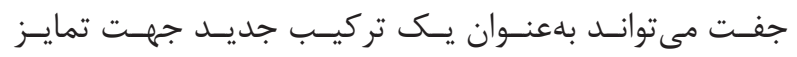

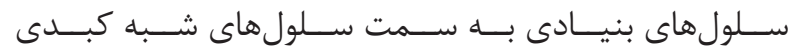

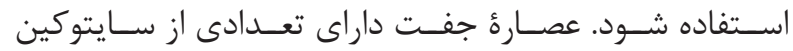

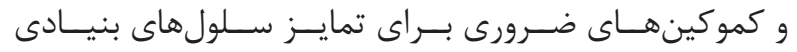

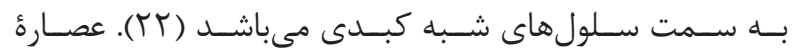

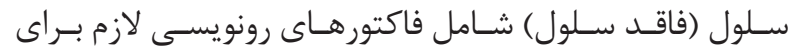

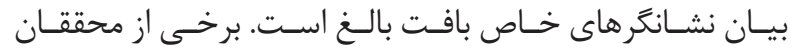

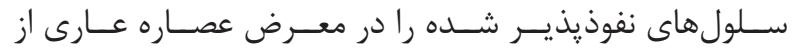

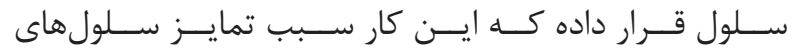

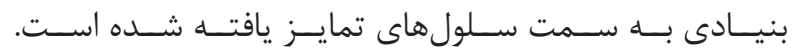

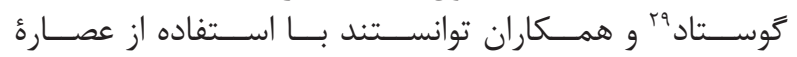

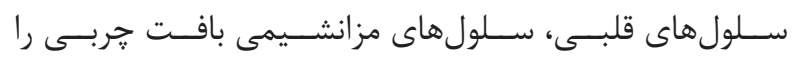

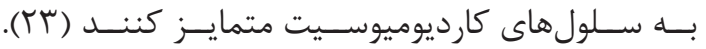

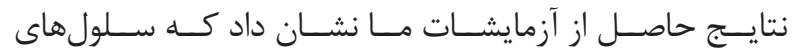

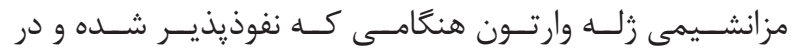

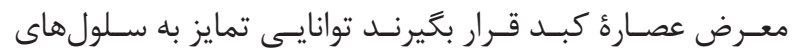

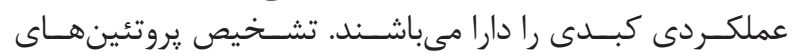

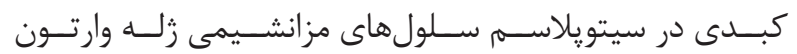

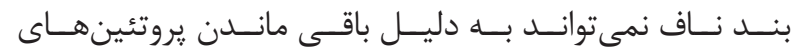

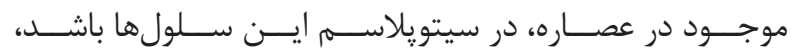

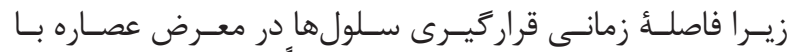

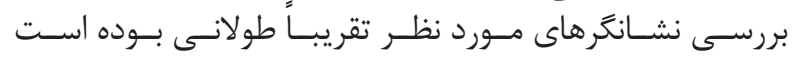

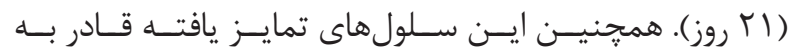

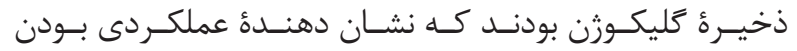

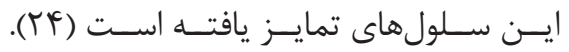

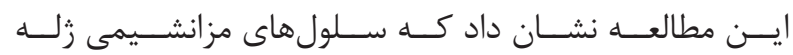

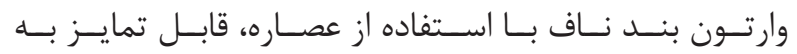

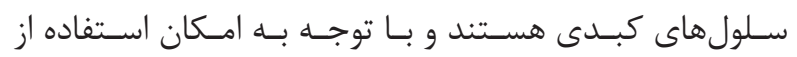

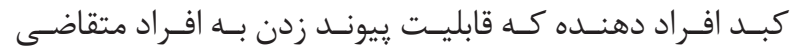

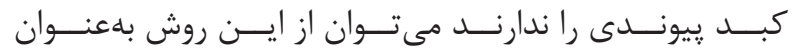

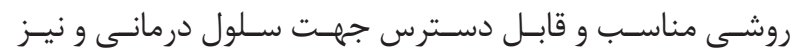

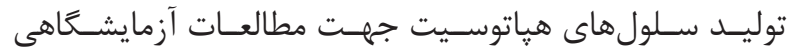

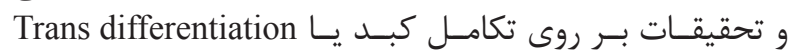

$$
\text { اسـتفاده كـرد. }
$$

1. Guettier C. Which stem cells for adult liver? Ann Pathol. 2005; 25(1): 33-44.

2. Klingemann H, Matzilevich D, Marchand J. Mesenchymal stem cells-sources and clinical applications. Transfus Med Hemother. 2008; 35(4): 272-7.

3. Salem HK, Thiemermann C. Mesenchymal stromal cells: current understanding and clinical status. Stem Cells. 2010; 28(3): 585-96.

4. Wells WA. Is transdifferentiation in trouble? J Cell

26 Trans differentiation

${ }^{27}$ Autologous

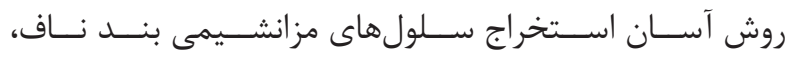

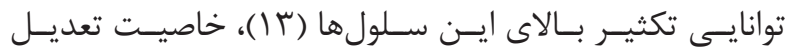

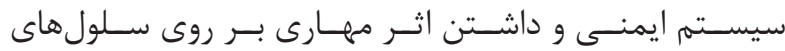

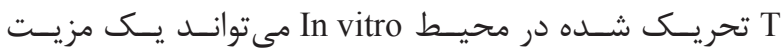

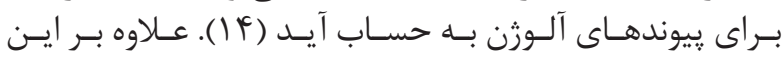

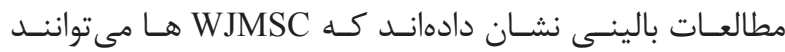

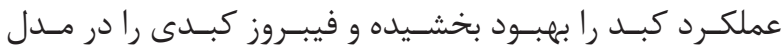

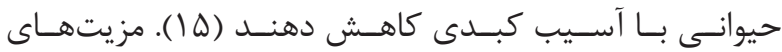

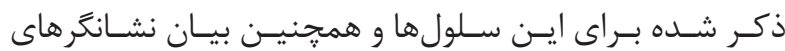

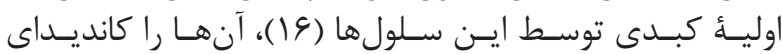

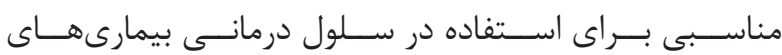

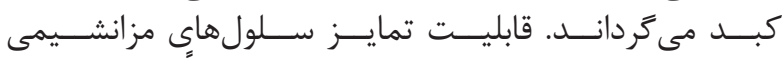

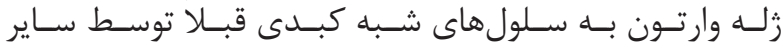

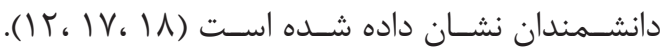

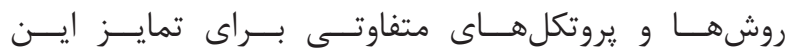

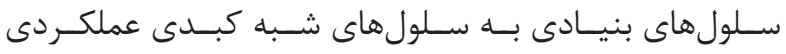

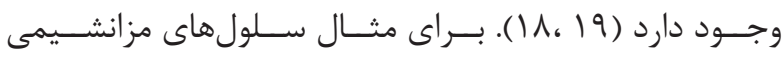

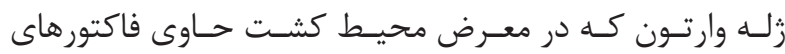

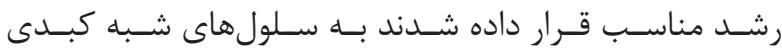

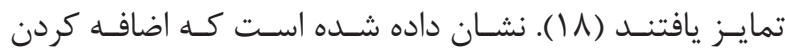

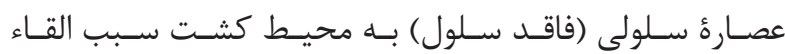

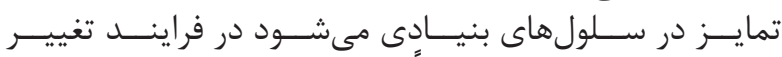

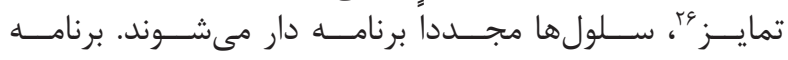

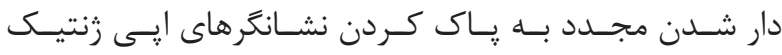

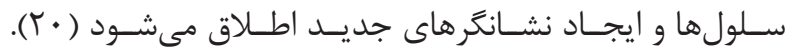

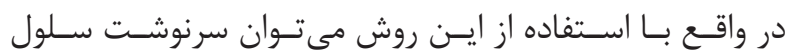

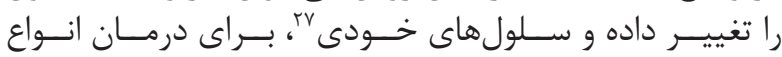

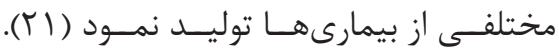

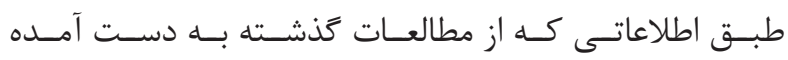

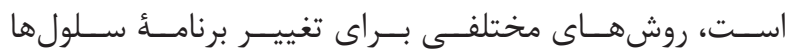

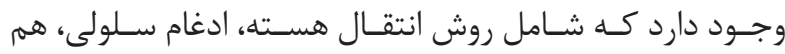

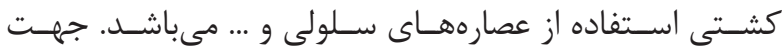

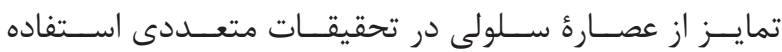

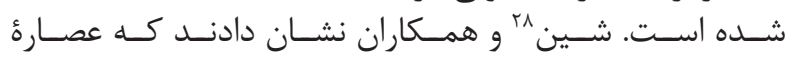

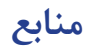

Biol. 2002; 157(1): 15-8.

5. Wilmut I, Beaujean N, De Sousa P, Dinnyes A, King T, Paterson L, et al. Somatic cell nuclear transfer. Nature. 2002; 419(6907): 583-7.

6. Matsumura H, Tada T. Cell fusion-mediated nuclear reprogramming of somatic cells. Reprod Biomed Online. 2008; 16(1): 51-6.

7. Park IH, Zhao R, West JA, Yabuuchi A, Huo H, Ince $\mathrm{TA}$, et al. Reprogramming of human somatic cells

\footnotetext{
${ }^{28}$ Shin

${ }^{29}$ Gaustad
} 
to pluripotency with defined factors. Nature. 2007; 451(7175): 141-6.

8. Burguera EF, Bitar M, Bruinink A. Novel in vitro coculture methodology to investigate heterotypic cell-cell interactions. Eur Cell Mater. 2010; 19: 166-79.

9. Collas P, Taranger CK. Epigenetic reprogramming of nuclei using cell extracts. Stem Cell Rev. 2006; 2(4): 309-17.

10. Chao KC, Chao KF, Fu YS, Liu SH. Islet-like clusters derived from mesenchymal stem cells in Wharton's Jelly of the human umbilical cord for transplantation to control type 1 diabetes. PLoS One. 2008; 3(1): e1451. doi: 10.1371/journal.pone.0001451.

11. Mitchell KE, Weiss ML, Mitchell BM, Martin P, Davis D, Morales L, et al. Matrix cells from Wharton's jelly form neurons and glia. Stem Cells. 2003; 21(1): 50-60.

12. Borhani-Haghighi M, Talaei-Khozani T, Ayatollahi M, Vojdani Z. Wharton's jelly-derived mesenchymal stem cells can differentiate into hepatocyte-like cells by HepG2 cell line extract. Iranian Journal of Medical Sciences. 2015; 40(2): 143-51.

13. Anzalone R, Iacono ML, Corrao S, Magno F, Loria T, Cappello F, et al. New emerging potentials for human Wharton's jelly mesenchymal stem cells: immunological features and hepatocyte-like differentiative capacity. Stem Cells Dev. 2010; 19(4): 423-38.

14. Weiss ML, Anderson C, Medicetty S, Seshareddy KB, Weiss RJ, Vander Werff I, et al. Immune properties of human umbilical cord wharton's jelly-derived cells. Stem Cells. 2008; 26(11): 2865-74.

15. Yan Y, Xu W, Qian H, Si Y, Zhu W, Cao H, et al Mesenchymal stem cells from human umbilical cords ameliorate mouse hepatic injury in vivo. Liver Int. 2009; 29(3): 356-65.
16. Zhang S, Chen L, Liu T, Zhang B, Xiang D, Wang Z, et al. Human umbilical cord matrix stem cells efficiently rescue acute liver failure through paracrine effects rather than hepatic differentiation. Tissue Eng Part A. 2012; 18(13-14): 1352-64.

17. Zhao Q, Ren H, Li X, Chen Z, Zhang X, Gong $\mathrm{W}$, et al. Differentiation of human umbilical cord mesenchymal stromal cells into low immunogenic hepatocyte-like cells. Cytotherapy. 2009; 11(4): 414-26.

18. Talaei-Khozani T, Borhani-Haghighi M, Ayatollahi M, Vojdani Z. An in vitro model for hepatocyte-like cell differentiation from Wharton's jelly derivedmesenchymal stem cells by cell-base aggregates. Gastroenterology and Hepatology from Bed to Bench. 2015; 8(3): 188

19. Ayatollahi M, Soleimani M, Tabei SZ, Salmani MK. Hepatogenic differentiation of mesenchymal stem cells induced by insulin like growth factor-I. World J Stem Cells. 2011; 3(12): 113-21.

20. Hochedlinger K, Jaenisch R. Nuclear reprogramming and pluripotency. Nature. 2006; 441 (7097): 1061-7.

21. Gurdon JB, Melton DA. Nuclear reprogramming in cells. Science. 2008; 322(5909): 1811-5.

22. Shin K, Lee H, Jung J, Cha D, Kim G. Culture and in vitro hepatogenic differentiation of placenta-derived stem cells, using placental extract as an alternative to serum. Cell Prolif. 2010; 43(5): 435-44.

23. Gaustad KG, Boquest AC, Anderson BE, Gerdes AM, Collas P. Differentiation of human adipose tissue stem cells using extracts of rat cardiomyocytes. Biochem Biophys Res Commun. 2004; 314(2): 420-7.

24. Yamada T, Yoshikawa M, Kanda S, Kato Y, Nakajima $\mathrm{Y}$, Ishizaka $\mathrm{S}$, et al. In vitro differentiation of embryonic stem cells into hepatocyte-like cells identified by cellular uptake of indocyanine green. Stem Cells. 2002; 20(2): 146-54. 